\title{
DUAL-POLARIZED ANTENNA SUB-ARRAY OF $2 \times 2$ ELEMENTS FOR MEASUREMENTS OF SHORT ELECTROMAGNETIC PULSES
}

\author{
Evgeny Balzovsky, Yury Buyanov, Vladimir Kosheleva, Eduard Nekrasov \\ Institute of High Current Electronics SB RAS, 634055, Tomsk, Russia
}

\begin{abstract}
Results of the investigations of a dual-polarized antenna module of a $2 \times 2$ receiving antenna array designed for recording the waveform of short electromagnetic pulses are presented. Each element of the antenna array is an active dipole that is loaded on the single-stage FETbased amplifier. Results of the measurements of the patterns, effective length, and waveforms of the recorded pulses are presented.
\end{abstract}

\section{Introduction}

In the tasks of the detection of people behind optically opaque obstacles using ultrawideband (UWB) radiation [1, 2], as well as in studying the electric field structure of short electromagnetic pulses, it is important that the recorded waveforms of the pulses would not have significant distortions. Decrease of the spatial length of the radiation pulses results in the increase of the resolution of the sounded objects. At the same time, a high frequencies in the spectrum of short pulses are intensively absorbed in the obstacle material or in the lossy medium under study. The range of the optimal length of bipolar voltage pulses exciting the transmitting antenna is $1-4 \mathrm{~ns}$. It corresponds to the frequency range of 0.15-2 GHz. To register UWB pulses with a spectrum in this range, receive antennas and antenna arrays are required with the waveform of the voltage at the output of which is proportional to the electric field strength.

The use of UWB pulses allows not only providing high spatial resolution, but also solving the problems of recognition of sounded objects by changing the waveform of the reflected electromagnetic field [3]. In this case, a significant amount of information is contained in the polarization structure of pulses reflected from objects. The elements of the dual-polarized receiving array should register two orthogonal components of the pulsed field. In [4], active antennas consisting of two perpendicular dipoles with aligned centers are suggested. These dipoles allow recording UWB pulses with a spectrum in the frequency range of $0.4-3.5 \mathrm{GHz}$. However the proposed in [4] antennas have a balancing outputs. This is a disadvantage when combining such dipoles into the antenna array, since it is necessary to use a complex beam-forming circuit and an external UWB balancing-tounbalancing network (balun). In [5], a lower-frequency active dipole antenna with a bandwidth of $0.15-2 \mathrm{GHz}$ is proposed. Due to the use of a miniature built-in UWB balun,

${ }^{\text {a }}$ Corresponding author: koshelev@lhfe.hcei.tsc.ru 
this antenna has an asymmetrical output. Since it is difficult to align the centers of such dipoles for recording the orthogonal field components, so the dipoles of vertical and horizontal polarization are shifted by half step of the array relative to each other.

One of the possible schemes of UWB radar can be radiation of objects from one source and electronic steering by a receiving array. To control the array pattern, the signals from each antenna element are summing together with the necessary time shifts. With an increase in the number of elements, the signal summation circuit becomes the key element of the antenna array. The fidelity of the waveform at the array output depends on the frequency characteristics of the summation circuit. It is suggested to use a binary scheme for signal summing which can be realized using multistage ring-type power combiners.

To reveal the features of the construction of the antenna array with a large number of elements, a dual-polarized module consisting of eight dipoles was developed and investigated presenting two sub-arrays of $2 \times 2$ dipoles recording two orthogonal components of the short-pulse electric field. The design of sub-array module and the summation unit, as well as the results of research of their characteristics, are described below.

\section{Antenna module design}

Figure 1 presents the antenna array module physical configuration. The active dipoles 1 form a $2 \times 2$ sub-array of the vertical channel, the dipoles 2 form a $2 \times 2$ sub-array of the horizontal channel. The feeder lines are covered up with the bulk absorbent material 3 to reduce the effect of reflections from the summation unit 4 located on the rear side of array. The $2 \times 2 \times 2$ antenna module has outputs 5 for connection to two channels of the oscilloscope. For dipoles of one channel, the distance between the centers is $67 \mathrm{~mm}$, and the distance between the nearest ends of the dipole arms is $6.7 \mathrm{~mm}$.

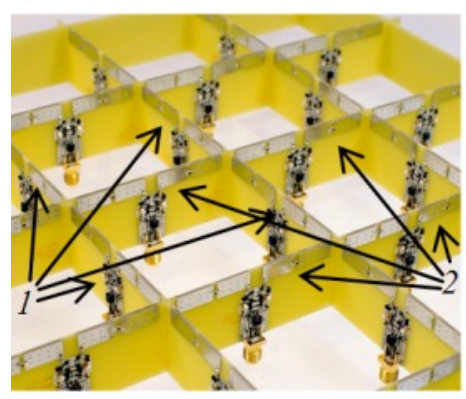

a)

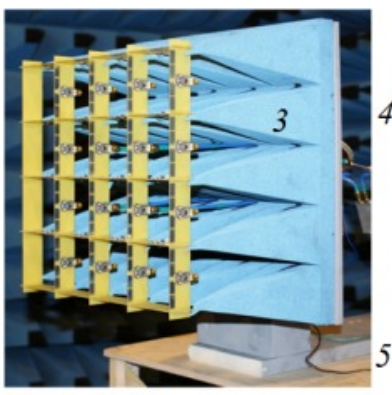

b)

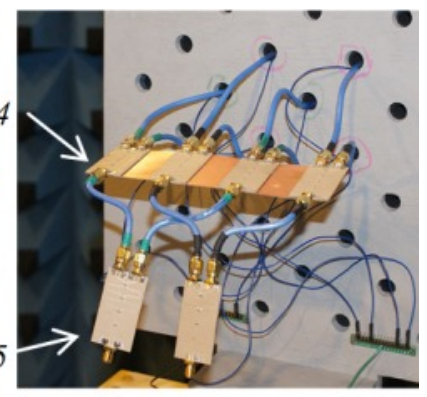

c)

Fig. 1. Module of a $2 \times 2 \times 2$ element in the antenna array: a) layout of elements; b) side view; c) back view. 1 - vertical channel dipoles; 2 - horizontal channel dipoles; 3 - volume absorber material; 4 - the stage of summation circuit; 5 - output connectors of vertical and horizontal channels.

The construction of the dipole is analogous to that given in [5]. The difference is the implementation of printed-circuit board on double-sided material with metallized through holes. The active dipole (see Figure 2) is made on a 1-mm thick FR4 plate with size of $60 \times 27 \mathrm{~mm}$. The arms 1 and 2 of the dipole are connected to the balancing active FET-based element 3 which is connected to the output connector 5 via the ferrite broadband balun 4 . 


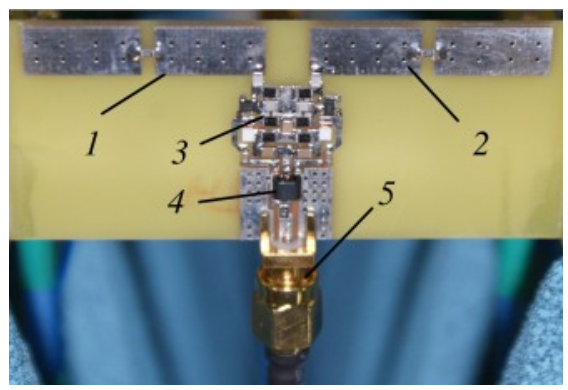

Fig. 2. Appearance of the antenna element. 1 and 2 - dipole arms; 3 - active element; 4 - balun; 5 - output connector.

\section{Ultrawideband summation unit}

\subsection{Design of the summation unit}

To reduce the signal attenuation and to increase the decoupling between channels, a circuit based on the ring power combiners was developed and implemented. To extend the bandwidth, the summation unit consists of several stages. It is known that with increasing the number of stage the wave impedance of the near-to-output stage should increase. However, the implementation of a stripline with high wave impedance is related to the limitations of the printing circuit board technology, which does not allow making too narrow conductors. Taking into account the minimum possible width of the conductor, a four-stage summation unit made on a 1-mm thick foil-clad dielectric FLAN having a relative permittivity of 10 was chosen.

Calculations of the summation unit were performed in the approximation of cascade connection of the four-terminal devices. Figure 3 presents the physical configuration of the summation unit, where connectors 1 and 2 are the inputs, and connector 3 is the output. The wave impedances of the line segments L1-L4 are 56, 64, 76 and $85.5 \mathrm{Ohms}$, respectively. The width of the L4 line is $0.2 \mathrm{~mm}$. The choice of nominal values of the surface-mount resistors is limited by the standard E24 series. The nominal values of the resistors R1-R4 of the standard size 0603 are 470, 300, 160 and $100 \mathrm{Ohms}$, respectively. The size of the dielectric plate is $46.5 \times 25.7 \mathrm{~mm}$.

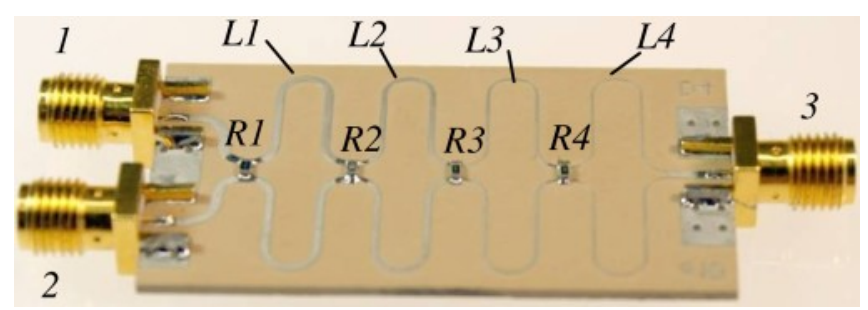

Fig. 3. Physical configuration of the summation unit; 1 and 2 -inputs; 3 - output.

\subsection{Characteristics of the summation unit}

Figure $4 a$ illustrates the voltage standing-wave ratio (VSWR) of the summation unit calculated analytically (curve 1), obtained as a result of full-wave simulation (curve 2) and measurements (curve 3). The VSWR of the inputs is no higher than 1.5 in the range of 0.1-2.2 GHz. Figure $4 b$ shows the frequency dependence of transmission coefficient 
module $\left|S_{31}\right|$ of the summing unit. At the central frequency, measured $\left|S_{31}\right|=3.3 \mathrm{~dB}$. The deviation of the values of the transmission coefficient module is no higher than $0.4 \mathrm{~dB}$ in the frequency range of $0.1-2.2 \mathrm{GHz}$. In this range, the phase-frequency characteristic differs from the linear dependence by no more than 10 degrees. The measured isolation between the inputs 1 and 2 in the frequency range of $0.17-2 \mathrm{GHz}$ exceeds $20 \mathrm{~dB}$, and in the frequency ranges of $0.1-0.17 \mathrm{GHz}$ and $2-2.2 \mathrm{GHz}$ it exceeds $15 \mathrm{~dB}$. The developed UWB summation unit is suitable for synchronous addition of short pulses with a spectrum in the frequency band of $0.1-2.2 \mathrm{GHz}$ with low losses and distortions.

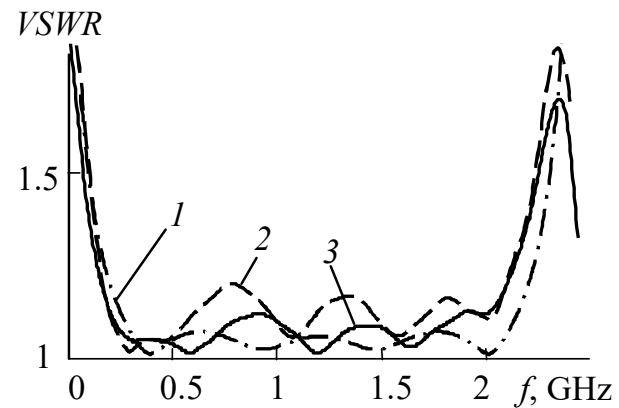

a)

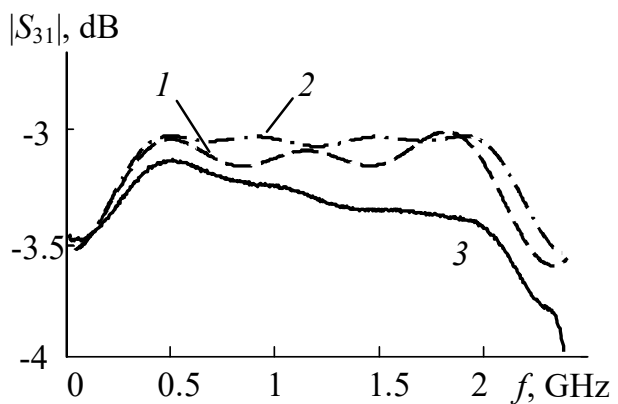

b)

Fig. 4. VSWR $(a)$ and transmission coefficient module $(b)$ of the summation unit. 1 - analytical calculation, 2 - full wave simulation, 3 - results of measurements.

\section{Antenna module characteristics}

In the time domain the characteristics of the antenna array module are measured by using a set of generators of short UWB pulses and a set of combined antennas [3], optimized for the effective radiation of pulses with different spectra. Figure 5 shows the waveforms of the voltage pulses $V(t)$ at the output of the receiving antenna array module (curves 1). Curves 2 correspond to the pulses at the output of the TEM-antenna. The spectra (by the level of 0.1 from the maximum value) of the pulses radiated by the antenna occupy the frequency bands of $10 \mathrm{MHz}-1 \mathrm{GHz}, 50 \mathrm{MHz}-1.3 \mathrm{GHz}, 80 \mathrm{MHz}-2.2 \mathrm{GHz}$, and $250 \mathrm{MHz}-4.3 \mathrm{GHz}$.

A TEM-antenna with a ground plate size of $120 \times 50 \mathrm{~cm}$ and an aperture height of $8 \mathrm{~cm}$ was used as a reference antenna for measuring the waveform of UWB pulses. The voltage at the output of the TEM-antenna is proportional to the electric field strength of the incident field, and the effective length is equal to half the height of the aperture in a wide range and has no dependence on the frequency. To quantify the distortion of the waveform of the pulses, the root-mean-square (RMS) deviation $\sigma$ of the voltage pulse waveform $V(t)$ at the output of the antenna array and the voltage $U(t)$ at the output of the reference antenna were calculated according to the following expression: $\sigma=\sqrt{\int_{T}\left[u\left(t-t_{0}\right)-v(t)\right]^{2} d t / \int_{T} u^{2}(t) d t}, \quad$ where $\quad u(t)=U(t) / \sqrt{\int_{T} U^{2}\left(t^{\prime}\right) d t^{\prime}} \quad$ and $v(t)=V(t) / \sqrt{\int_{T} V^{2}\left(t^{\prime}\right) d t^{\prime}}$ are the normalized functions, $T$ is the time window, and $t_{0}$ is the time shift of $U(t)$ with respect to $V(t)$, at which $\sigma$ takes the minimum value. The RMS deviation of the waveform of the pulses at the output of the array module from the pulses at the output of the TEM-antenna is shown in Table 1. The effective length of the antenna array calculated as the product of the effective length of TEM-antenna (it is equal to $4 \mathrm{~cm}$ ) by the ratio of the peak voltage at the antenna array module output to the peak voltage at the output of the TEM-antenna is presented in Table 1 as well. 

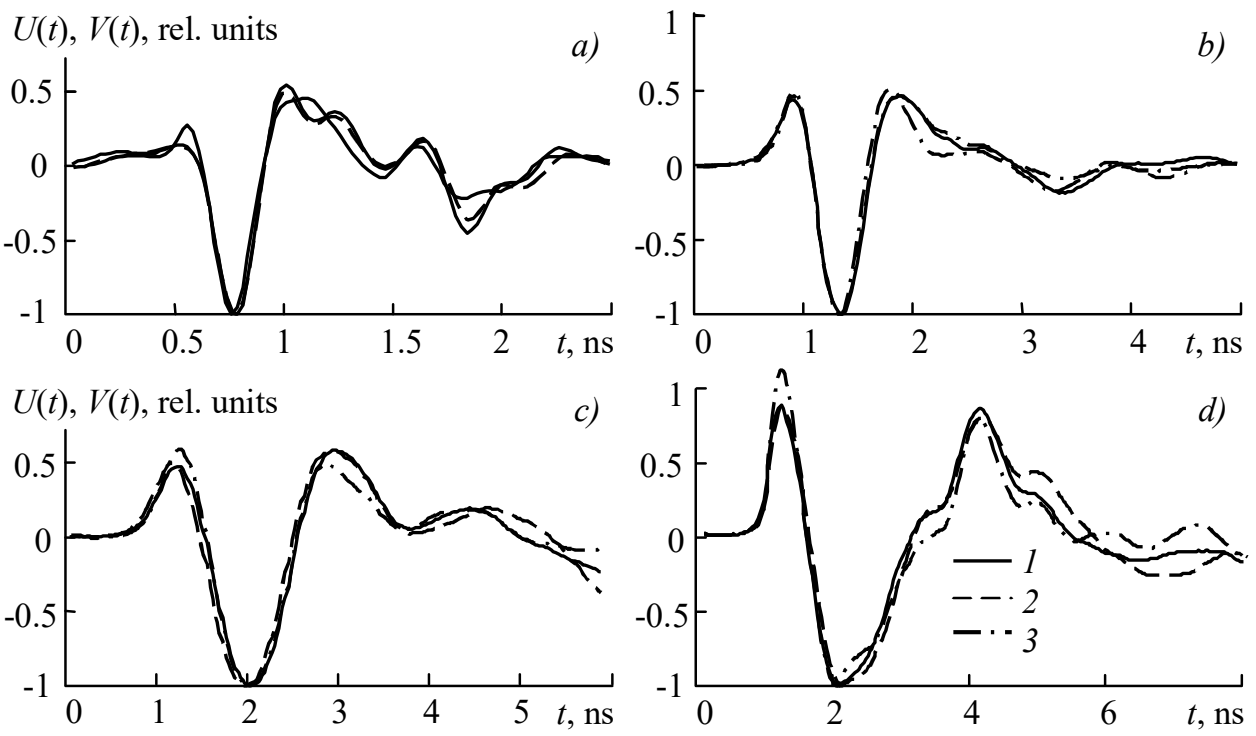

Fig. 5. Pulse waveforms at the antenna module output at the excitation of transmitting antennas by bipolar pulses of the length $0.5 \mathrm{~ns}$ (a); $1 \mathrm{~ns}$ (b), $2 \mathrm{~ns}$ (c), and $3 \mathrm{~ns}$ (d). 1 - horizontal channel of the antenna array module; 2 - vertical channel of the antenna array module; 3 - TEM-antenna.

Table 1. Characteristics of the antenna module channels

\begin{tabular}{|c|c|c|c|c|}
\hline \multirow{2}{*}{$\begin{array}{c}\text { Duration of pulse of } \\
\text { generator, ns }\end{array}$} & \multicolumn{2}{|c|}{$\sigma$} & \multicolumn{2}{c|}{ Efective length, cm } \\
\cline { 2 - 5 } & $\begin{array}{c}\text { Vertical } \\
\text { channel }\end{array}$ & $\begin{array}{c}\text { Horizontal } \\
\text { channel }\end{array}$ & $\begin{array}{c}\text { Vertical } \\
\text { channel }\end{array}$ & $\begin{array}{c}\text { Horizontal } \\
\text { channel }\end{array}$ \\
\hline 0.5 & 0.24 & 0.23 & 3.7 & 3.9 \\
\hline 1 & 0.19 & 0.21 & 4.5 & 4.5 \\
\hline 2 & 0.2 & 0.14 & 5.5 & 5.6 \\
\hline 3 & 0.24 & 0.2 & 5.7 & 6.0 \\
\hline
\end{tabular}

Figure 6 presents the measured patterns of the antenna module in two planes. Here, the pattern is the angular dependence of the value of voltage maximum at the output of the antenna array during the pulse.
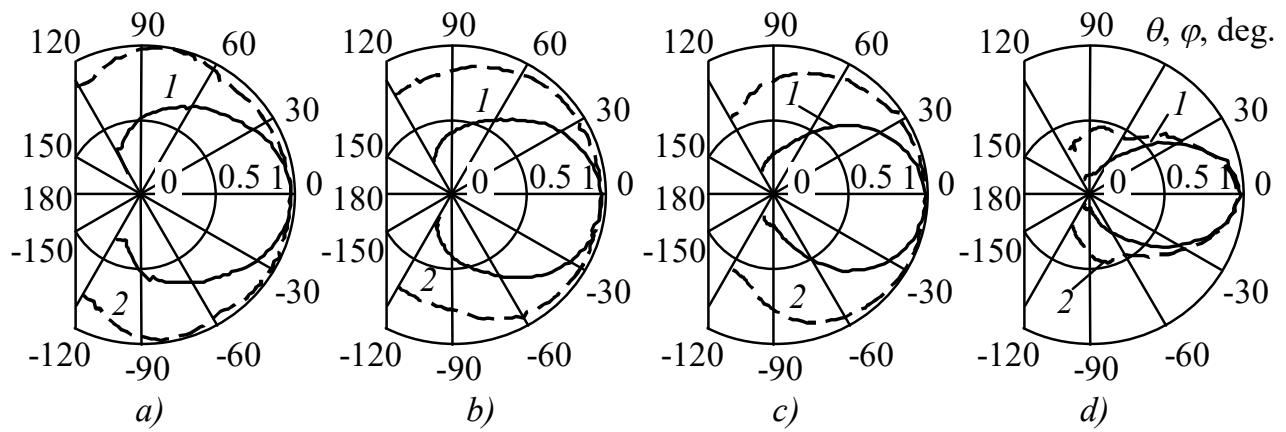

Fig. 6. Patterns of the antenna module at the excitation of the transmitting antennas by bipolar pulses of the duration of $3 \mathrm{~ns}(a), 2 \mathrm{~ns}(b), 1 \mathrm{~ns}(c)$, and $0.5 \mathrm{~ns}(d)$. Curves 1 correspond to the $E$-plane pattern, and curves 2 correspond to the $H$-plane pattern. 


\section{Conclusion}

The dual-polarized antenna module of a $2 \times 2$ elements of receiving antenna array designed for recording the temporal waveform of short electromagnetic pulses with a spectrum in the frequency range of $0.15-2 \mathrm{GHz}$ was developed and investigated. In the circuit of signal adding from each antenna element, it is provided to connect the controlled delay lines both at the output of each element for steering in a solid angle, and at the outputs of columns or the array lines for steering in the plane. The antenna module was tested by means of shortlength (1-4 ns) UWB pulses. The temporal waveform of the voltage pulses at the output of the developed antenna array and at the output of the reference TEM-antenna differ by no more than $20 \%$ by the root mean square criterion. When exposed to subnanosecond pulses, in which the upper frequency in the spectrum exceeds $4 \mathrm{GHz}$, the distortion of the pulse waveform is no higher than $25 \%$ as compared to the TEM-antenna. The aim of the further work is to develop an antenna array with a large number of elements.

\section{Acknowledgements}

The work was supported by the Russian Science Foundation, project No. 16-19-10081.

\section{References}

[1] E. Balzovsky, Yu. Buyanov, MATEC Web of Conferences 79, 01036 (2016) doi: 10.1051/matecconf $/ 20167901036$

[2] S. Shipilov, R. Satarov, I. Fedyanin, E. Balzovsky, V. Yakubov, MATEC Web of Conferences 79, 01079 (2016) doi: 10.1051/matecconf/20167901079

[3] V. Koshelev, Yu. Buyanov, V. Belichenko, Ultrawideband Short-Pulse Radio Systems (Artech House, Norwood, MA, 2017)

[4] E. Balzovskii, Yu. Buyanov, V. Koshelev, J. Commun. Technol. Electron. 55, 172 (2010) doi: 10.1134/S1064226910020087

[5] E. Balzovsky, Yu. Buyanov, V. Koshelev, E. Nekrasov. IOP J. Phys: Conf. Series 881, 012001 (2017) doi: 10.1088/1742-6596/881/1/012001 